\title{
Phonon Density of States and Compression Behavior in Iron Sulfide under Pressure
}

\author{
Hisao Kobayashi* \\ Graduate School of Material Science, University of Hyogo, 3-2-1 Koto Hyogo 678-1297, Japan \\ and Department of Physics, Tohoku University, Sendai, 980-8578, Japan \\ Takashi Kamimura \\ Department of Physics, Tohoku University, Sendai, 980-8578, Japan \\ Dario Alfè \\ Department of Earth Sciences and Department of Physics and Astronomy, University of College London, \\ London WCIE 6BT, United Kingdom \\ Wolfgang Sturhahn, Jiyong Zhao, and Esen E. Alp \\ Experimental Facilities Division, Argonne National Laboratory, Argonne, Illinois 60439, USA
}

(Received 23 March 2004; published 4 November 2004)

\begin{abstract}
We report the partial phonon densities of states (DOS) of iron sulfide, a possible component of the rocky planet's core, measured by the ${ }^{57} \mathrm{Fe}$ nuclear resonant inelastic $\mathrm{x}$-ray scattering and calculate the total phonon DOS under pressure. From the phonon DOS, we drive thermodynamic parameters. A comparison of the observed and estimated compressibilities makes it clear that there is a large pure electronic contribution in the observed compressibility in the metallic state. Our results present the observation of thermodynamic parameters of iron sulfide with the low-spin state of an $\mathrm{Fe}^{2+}$ ion at the high density, which is similar to the condition of the Martian core.
\end{abstract}

DOI: $10.1103 /$ PhysRevLett.93.195503

PACS numbers: 63.20.-e, 71.30.+h, 76.80.+y, 91.60.Gf

Iron sulfide, $\mathrm{FeS}$, has been attracting attention in geosciences as well as condensed matter physics. The crystallographic properties of $\mathrm{FeS}$ have been investigated extensively at high pressures and/or high temperatures by $\mathrm{x}$-ray diffraction measurements [1-5] because it is believed to be a component of the core of rocky planets such as Earth and Mars. Although the pressure vs temperature phase diagram has been contradictory, FeS undergoes two successive first-order phase transitions at room temperature, from the troilite $(P \overline{6} 2 c)$ to a MnP-type (Pnma) structure at $3.5 \mathrm{GPa}$ and then to a monoclinic structure at $6.5 \mathrm{GPa}$ with about $7 \%$ volume reduction. The compressibilities in those three phases were measured to be $13.6,17.5$, and $7.8 \times 10^{-3} \mathrm{GPa}^{-1}$, respectively. The thermodynamic properties under pressure are essential for understanding the core materials of the planets. However, there is no experimental knowledge of the lattice dynamics in FeS under pressure. Phonon dispersion relations and phonon densities of states (DOS) characterize the thermodynamic properties of the materials.

At ambient conditions, $\mathrm{FeS}$ is an antiferromagnetic semiconductor with $T_{\mathrm{N}}=589 \mathrm{~K}$ and belongs to the charge-transfer regime [6,7], where an electron correlation plays an important role. Electrical resistivity measurements of $\mathrm{FeS}$ under pressure [8] show that the semiconductor-metal and metal-semiconductor transitions occur at 3.5 and $6.5 \mathrm{GPa}$, respectively, corresponding to the structural phase transitions at room temperature. The energy gap in the semiconductor state below $3.5 \mathrm{GPa}$ is caused by the electronic correlation, while the gap opens up between the nonbonding and antibonding bands in the semiconductor above $6.5 \mathrm{GPa}$. These phase transitions cause simultaneous changes in the electronic and structural properties. Therefore, we need to investigate the phonon properties of $\mathrm{FeS}$ in each phase and around these phase transitions from both the geophysics and condensed matter physical points of view. A new method based on nuclear resonant inelastic $\mathrm{x}$-ray scattering (NRIXS) was introduced to measure partial phonon DOS in samples containing suitable Mössbauer nuclei $[9,10]$. Recently, this technique was successfully applied to measure the phonon DOS of $\varepsilon$-Fe under pressure $[11,12]$.

In this Letter, we have applied this new method to extract the partial phonon DOS of FeS under pressure and then calculated the total phonon DOS. It is found that the phonon DOS of FeS is modified by the pressureinduced phase transitions, and we derive the pressure dependence of thermodynamic parameters from these phonon DOS. In these thermodynamic parameters, the pressure dependence of a force constant is determined by Hund's rules of $\mathrm{Fe}^{2+}$ ion in $\mathrm{FeS}$. We evaluate the pure electronic contribution in the compressibility of the metallic state.

The polycrystalline samples enriched with 50 at. \% ${ }^{57} \mathrm{Fe}$ were prepared by a method described elsewhere [8]. The obtained samples were confirmed to be in the single phase by $\mathrm{x}$-ray diffraction and have a stoichiometric composition within the experimental accuracy. Experiments under high pressure were performed on 
3-ID beam line at the Advanced Photon Source, Argonne National Laboratory using a special diamond anvil cell (DAC) up to $12 \mathrm{GPa}$ [12]. The pulsed synchrotron radiation was monochromatized with $2 \mathrm{meV}$ resolution by a high-resolution monochromator [13] and then focused on a $5 \times 5 \mu \mathrm{m}^{2}$ area of the sample in the DAC by two orthogonal Kirkpatrick-Baez mirrors. The ${ }^{57} \mathrm{Fe}$ NRIXS spectra were measured by tuning the highly monochromatized beam in an energy range of about $\pm 100 \mathrm{meV}$. Under high pressure, the powder samples were loaded with ruby crystals into a sample cavity of a $0.15 \mathrm{~mm}$ diameter in a $0.2 \mathrm{~mm}$ thick Be metal gasket. The use of Fluorinert as the pressure-transmitting medium ensured quasihydrostatic conditions. Pressure was calibrated by measuring the wavelength shift of the $R_{1}$ luminescence line of the ruby crystal. We have also measured the delaytime spectra of ${ }^{57} \mathrm{Fe}$ coherent nuclear forward scattering in $\mathrm{FeS}$ under pressure to monitor the phase transitions [14].

Figure 1 shows typical NRIXS spectra, which consist of large center peaks originating from elastic scattering and sidebands resulting from inelastic scattering with the annihilation and creation of phonons. The resolution is sufficient to resolve the modification of inelastic components in the observed spectra by the structural phase transitions as shown in Fig. 1. The inelastic components in the spectra extract lead to the partial phonon DOS assuming a harmonic lattice model [15]. The extracted partial phonon DOS are shown in Fig. 2. At 1.5 GPa in the

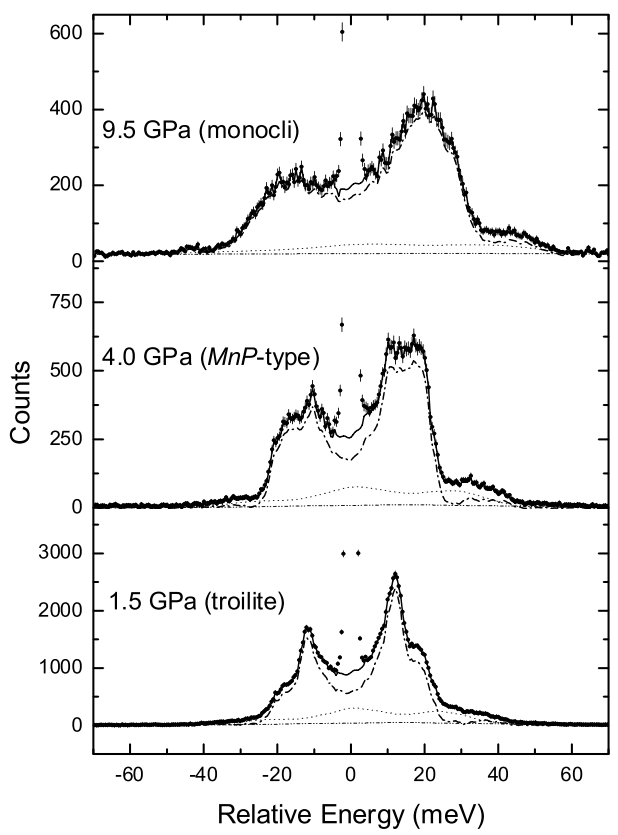

FIG. 1. Typical NRIXS spectra at 1.5, 4.0, and 9.5 GPa. The circles with error bars indicate the observed spectra and the solid lines represent the calculated inelastic components. The dash-dotted lines show the single phonon contribution subspectra and other two lines show two and multiphonon contribution subspectra. semiconductor with the troilite structure, there are two peaks at 12 and $18 \mathrm{meV}$ in the phonon DOS which probably originate from the transverse and longitudinal acoustic phonon branches. Furthermore, the highest-energy peak at $37 \mathrm{meV}$ is probably caused by the optical phonon branches. At 4.0 GPa in the metallic state with the MnPtype structure, there exists a strong peak at $20 \mathrm{meV}$ with a shoulder around $10 \mathrm{meV}$ in the phonon DOS. The spectrum of phonon DOS at $9.5 \mathrm{GPa}$ is shifted to higher energy because of the large volume reduction at 6.5 GPa. Since there are three different Fe sites in this unit cell [5], the spectrum has a more complicated structure than in the other two phases.

$A b$ initio calculations based on density-functional theory (DFT) in the generalized-gradient approximation were performed to determine the total phonon DOS of $\mathrm{FeS}$ at the same pressures using the projector augmented wave approach. Calculations were performed using the VASP code [16]. We calculated the force constant matrix and then the phonon frequencies of FeS with the three structures using our implementation of the small displacement method [17]. It was found that the troilite structure is unstable in the present calculation. Since $\mathrm{FeS}$ in the troilite structure is the semiconductor due to electron correlation, it is possible that this instability is due to the well known inaccurate DFT description of

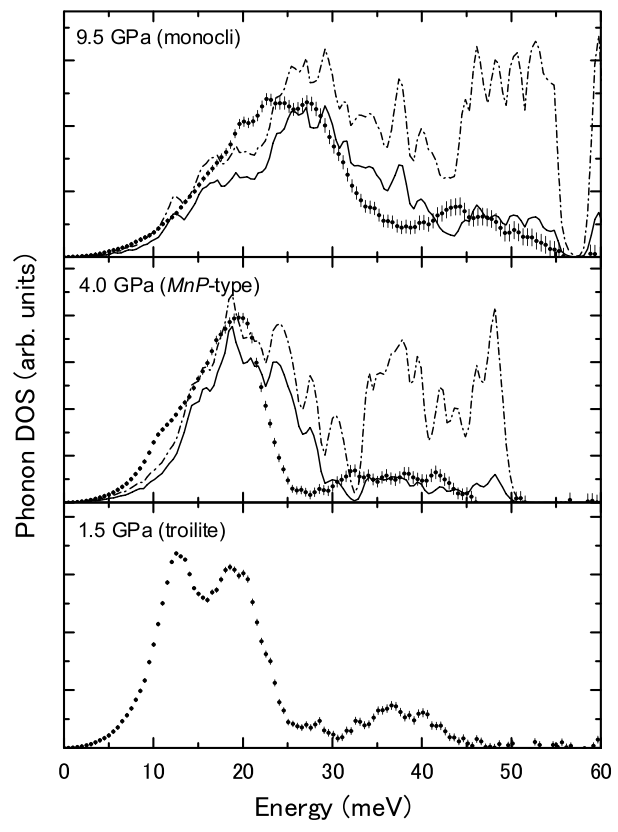

FIG. 2. Phonon DOS of FeS. The circles with error bars represent the partial phonon DOS extracted from NRIXS spectra at 1.5, 4.0, and $9.5 \mathrm{GPa}$. The dash-dotted and solid lines indicate the calculated total and partial phonon DOS, respectively. The partial phonon DOS is the measured Fe phonon DOS, and the total theoretical phonon DOS is the sum of $\mathrm{Fe}$ and $\mathrm{S}$. The calculated $\mathrm{Fe}$ phonon DOS agrees reasonably well both in terms of energy scale, as well as absolute magnitude. 
strongly correlated systems. We used supercells containing 128 and 24 atoms for the MnP-type and the monoclinic structures, respectively. The calculated phonon DOS spectra are shown in Fig. 2. The phonon modes related to $\mathrm{S}$ atoms are mainly above $30 \mathrm{meV}$, although the projection of the total phonon DOS onto $\mathrm{S}$ atoms is never zero even at the low energy region. The largest peak in each calculated partial phonon DOS qualitatively corresponds to that in the extracted partial phonon DOS within the experimental resolution. In the MnP-type phase, the calculated phonon DOS in the region from the peak to about $+10 \mathrm{meV}$ is enhanced over the measurement. This discrepancy originates in the difference between the experimental and the predicted volume and is reduced in the monoclinic phase.

We extract thermodynamic parameters by integration of phonon DOS with various energy weights and mean force constant from the experimentally measured phonon excitation probability, based on normalization sum rules as given by Lipkin [18] and Sturhahn et al. [10]. Above $3.5 \mathrm{GPa}$, the pressure dependences of the Fe components in these calculated thermodynamic parameters qualitatively agree with those obtained from the extracted partial phonon DOS as seen Fig. 3. These pressure dependences obtained from the experimental results show no discontinuity at $3.5 \mathrm{GPa}$ although about a 2 order decrease of magnitude in electrical resistivity was observed [8]. On the other hand, all thermodynamic parameters show distinct steplike features around 6.5 GPa.

First, we discuss the pressure dependence of the mean force constant $(\bar{D})$. In an ionic crystal, a short-range repulsive force determines the interatomic force constant. This short-range repulsive force arises from the charge distributions and Hund's rules of atoms. In a simple metal, there also exist interactions connecting a large number of ions in addition to this force because of a nonlocal electron ion potential. The measurement of ${ }^{57} \mathrm{Fe}$ Mössbauer spectra was carried out under pressure [19]. It was found that the pressure dependence of the center shift changes significantly at $6.5 \mathrm{GPa}$. This result indicated a substantial change of the $3 d$-electron configuration on the $\mathrm{Fe}$ ion at the second phase transition. Recently a Mössbauer study showed a nonmagnetic quadrupole spectrum down to $5 \mathrm{~K}$ at $12 \mathrm{GPa}$ and $\mathrm{x}$-ray emission experiments observed a pressure-induced reduction of the satellite amplitude in these spectra around 6.3 GPa [20,21]. These results strongly suggest the second phase transition is high spin to low spin (HS-LS) in the $\mathrm{Fe}^{2+}$ ion. Consequently, the distinct change in the pressure dependence of $\bar{D}_{\mathrm{Fe}}^{\mathrm{o}}$ around $6.5 \mathrm{GPa}$ is caused by the HSLS transition. Since $\bar{D}_{\mathrm{S}}^{\mathrm{c}}$ is larger than $\bar{D}_{\mathrm{Fe}}^{\mathrm{c}}$ above $3.5 \mathrm{GPa}$, the repulsive force range of the $\mathrm{S}$ atom exceeds the first nearest neighbor distance. The atomic radius of the $\mathrm{S}^{2-}$ ion is about twice as large as that of the $\mathrm{Fe}^{2+}$ ion and is comparable to half the S-S bond length in the three phases. The short-range repulsive force that acts on the

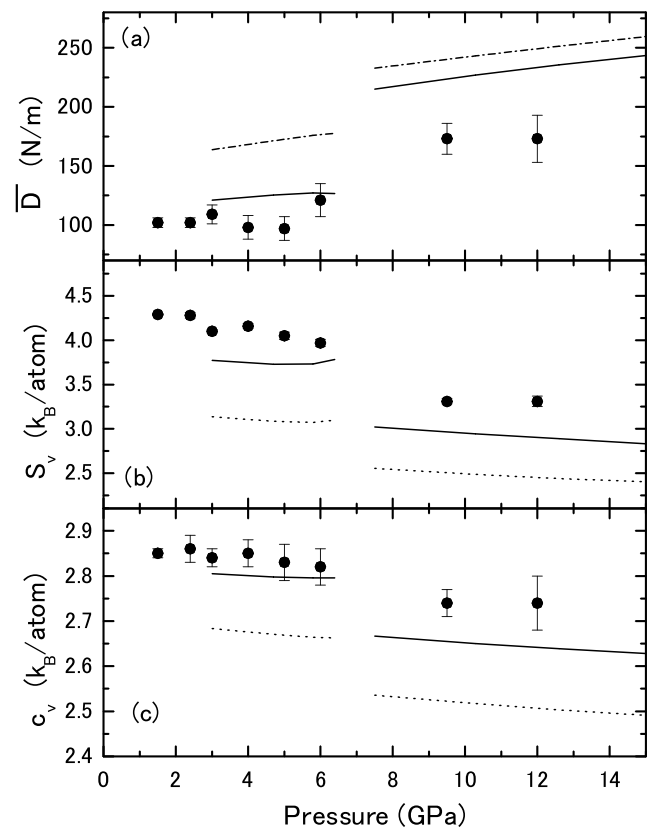

FIG. 3. Variation of the thermodynamical parameters estimated from the phonon DOS as a function of pressure: (a) mean force constant $(\bar{D})$ (b) entropy at constant volume $\left(S_{v}\right)$ and (c) specific heat at constant volume $\left(c_{v}\right)$. The circles with error bars represent $\bar{D}_{\mathrm{Fe}}^{\mathrm{o}}, S_{v}$, and $c_{v}$ evaluated from the extracted partial phonon DOS. The solid and dash-dotted lines in (a) indicate the calculated $\bar{D}$ for the $\mathrm{Fe}$ and $\mathrm{S}$ atoms, respectively. The broken and solid lines in (b) and (c) indicate $S_{v}$ and $c_{v}$ estimated from the calculated total and partial phonon DOS, respectively.

$\mathrm{S}$ atom originates from second nearest neighbor $\mathrm{S}$ atoms as well as from first nearest neighbor $\mathrm{Fe}$ atoms. Therefore, $\bar{D}_{\mathrm{Fe}}$ and $\bar{D}_{\mathrm{S}}$ are mainly given by the short-range repulsive force both in the semiconductor and metallic states in which the electronic structure is highly correlated. No significant anomaly is observed in the pressure dependence of $\bar{D}_{\mathrm{Fe}}^{\mathrm{o}}$ at $3.5 \mathrm{GPa}$

Second, we discuss the relation between $\bar{D}$ and the compressibility $\left(\kappa_{V}\right)$. The compressibility splits into two parts, one stemming from the interatomic force $\left(\kappa_{f c}\right)$ and the other one resulting from electronic contribution $\left(\kappa_{e}\right)$ which is the key parameter to describe the metalinsulator transition and the metallic state near the insulator phase [22]. In the homogeneous electron gas, $\kappa_{e}$ is defined as

$$
\kappa_{e}=\frac{1}{n^{2}} \frac{\partial n}{\partial \mu},
$$

where $n$ denotes electron density and $\mu$ denotes chemical potential. In the case where the short-range repulsive force acts between near neighbors [23], $\kappa_{f c}$ is expressed by

$$
\frac{1}{\kappa_{f c}} \propto \frac{M}{\sqrt[3]{v_{a}}} \sum_{i} \frac{\bar{D}_{i}}{2},
$$


where $v_{a}$ is the volume of a polyhedra, $M$ is a constant determined by the geometry of the polyhedra, and $i$ indicates an atom in the polyhedra. Thus $\kappa_{f c}$ is directly related to $\bar{D}$.

In the monoclinic phase, two of three different Fe sites have $\mathrm{S}$ atom coordination similar to the $\mathrm{MnP}$ structure although the structure is a more complicated one [5]. Even though we cannot estimate the exact value of $M$ in FeS, we assume in Eq. (2) that $M$ in the monoclinic phase is the same as in the MnP-type phase. Furthermore $v_{a}$ can be replaced by a volume of the chemical unit in the phases. Thus we evaluate the $\kappa_{V} / \kappa_{f c}$ ratios to be 0.68 and 0.50 for the MnP-type and the monoclinic phases, respectively, using $\bar{D}_{\mathrm{Fe}}^{\mathrm{c}}$ and $\bar{D}_{\mathrm{S}}^{\mathrm{c}}$. Since $\bar{D}_{\mathrm{Fe}}^{\mathrm{o}}$ shows no discontinuity at $3.5 \mathrm{GPa}$, we assume $\bar{D}_{\mathrm{Fe}}$ and $\bar{D}_{\mathrm{S}}$ in the troilite phase equal to those in the MnP-type phase. The ratio of the troilite phase is evaluated to be 0.46 . This ratio is in good agreement with that of the monoclinic phase in spite of the large difference in $\kappa_{V}$. Thus, this large difference originates from the interatomic force determined by the $3 d$-electron configuration of the Fe atoms in FeS, rather than electron correlations. Furthermore, the ratio in the metallic phase is much larger than those in the semiconductor phases. Since the electron-hole excitation with infinitely small energy occurs in a metallic state, the $\kappa_{e}$ value is usually finite. On the other hand, $\kappa_{e}$ can be neglected in a semiconductor state because the electron DOS vanishes at the Fermi energy. Consequently the larger $\kappa_{V}$ value in the metallic state is provided by a purely electronic contribution which is estimated to be about $40 \%$ of $\kappa_{V}$. Therefore, this discontinuous change of $\kappa_{e}$ characterizes the phase transition at $3.5 \mathrm{GPa}$.

Finally, the density of FeS in the monoclinic phase at room temperature is comparable to that of $\mathrm{FeS}$ with a hexagonal NiAs-type structure at the conditions of the Martian core, and recent $\mathrm{x}$-ray diffraction measurement indicates the monoclinic phase is stable up to $1225 \mathrm{~K}$ at $35 \mathrm{GPa}$ [24]. Since the value of $c / a$ in the NiAs-type phase shows an abrupt change at $6 \mathrm{GPa}$ and decreases below the ideal $c / a$ ratio of the hexagonal closed-packed structure [3], it is strongly suggested that the electronic state of the $\mathrm{Fe}$ atom in $\mathrm{FeS}$ is the low-spin one at the conditions of the Martian core. The vibrational parts of the thermodynamic properties are not free of the $3 d$-electronic configuration. Therefore, our thermodynamic data of $\mathrm{FeS}$ in the monoclinic phase give rise to a fundamental knowledge of physical states and a structural model of the Martian core.

We thank Dr. G. Shen and the GSE-CARS staff at the APS for their help with pressure calibration and Dr. H.-K. Mao of Carnegie Institute of Washington for providing drawings of their modified DAC. The work at Argonne is supported by U.S. DOE, BES Materials Science, under Contract No. W-31-109-ENG-38. H. K. acknowledges the support by the Japanese Ministry of Education, Culture, Sports, Science and Technology, Grant-in-Aid for
Scientific Research (c), 15540319. D. A. acknowledges support from the Royal Society and the Leverhulme Trust.

*Electronic address: kobayash@sci.u-hyogo.ac.jp

[1] H. E. King, Jr. and C. T. Prewitt, Acta Crystallogr. Sect. B 38, 1877 (1982).

[2] T. Kamimura, M. Sato, H. Takahashi, N. Môri, H. Yoshida, and T. Kaneko, J. Magn. Magn. Mater. 104107, 255 (1992).

[3] Y. Fei, C.T. Prewitt, H. K. Mao, and C. M. Bertka, Science 268, 1892 (1995).

[4] K. Kusaba, Y. Syono, T. Kikegawa, and O. Shimomura, J. Phys. Chem. Solids 58, 241 (1997).

[5] R. J. Nelmes, M. I. McMahon, S. A. Belmonte, and J. B. Parise, Phys. Rev. B 59, 9048 (1999).

[6] J. Zaanen, G. A. Sawatzky, and J.W. Allen, Phys. Rev. Lett. 55, 418 (1985); J. Magn. Magn. Mater. 54-57, 607 (1986).

[7] K. Shimada, T. Mizokawa, K. Mamiya, T. Saitoh, A. Fujimori, K. Ono, A. Kakizaki, T. Ishii, M. Shirai, and T. Kamimura, Phys. Rev. B 57, 8845 (1998).

[8] H. Kobayashi, N. Takeshita, N. Môri, H. Takahashi, and T. Kamimura, Phys. Rev. B 63, 115203 (2001).

[9] M. Seto, Y. Yoda, S. Kikuta, X.W. Zhang, and M. Ando, Phys. Rev. Lett. 74, 3828 (1995).

[10] W. Sturhahn, T. S. Toellner, E. E. Alp, X.W. Zhang, M. Ando, Y. Yoda, S. Kikuta, M. Seto, C.W. Kimball and B. Dabrowski, Phys. Rev. Lett. 74, 3832 (1995).

[11] R. Lübbers, H. G. Grünsteudel, A. I. Chumakov, and G. Wortmann, Science 287, 1250 (2000).

[12] H. K. Mao, J. Xu, V.V. Struzhkin, J. Shin, R. J. Hemly, W. Sturhahn, M. Y. Hu, E. E. Alp, L. Vocadlo, D. Alfé, G. D. Price, M. J. Gillan, M. Schwoerer-Böhning, D. Häusermann, P. Eng, G. Shen, H. Giefers, R. Lübbers, and G. Wortmann, Science 292, 914 (2001).

[13] T. S. Toellner, M. Y. Hu, W. Sturhahn, and E. E. Alp, Appl. Phys. Lett. 71, 2112 (1997).

[14] H. Kobayashi, Y. Yoda, and T. Kamimura, J. Phys. Soc. Jpn. 70, 1128 (2001).

[15] W. Sturhahn, Hyperfine Interact. 125, 149 (2000).

[16] G. Kresse and J. Furthmüller, Phys. Rev. B 54, 11169 (1996).

[17] D. Alfè, 1998, program available at http://chianti.geol.ucl.ac.uk/ dario

[18] H. J. Lipkin, Phys. Rev. B 52, 10073 (1995).

[19] H. Kobayashi, M. Sato, T. Kamimura, M. Sakai, H. Onodera, N. Kuroda, and Y. Yamaguchi, J. Phys. Condens. Matter 9, 515 (1997).

[20] S. Takele and G. R. Hearne, Phys. Rev. B 60, 4401 (1999).

[21] J.-P Rueff, C.-C. Kao, V.V. Struzhkin, J. Badro, J. Shu, R. J. Hemley, and H. K. Mao, Phys. Rev. Lett. 82, 3284 (1999).

[22] G. Kotliar, S. Murthy, and M. J. Rozenberg, Phys. Rev. Lett. 89, 046401 (2002).

[23] A. A. Maradudin, E.W. Montroll, G. H. Weiss, and I. P. Ipatova, Theory of Lattice Dynamics in the Harmonic Approximation (Academic Press, New York, 1971).

[24] A. Kavner, T. S. Duffy, and G. Shen, Earth Planet. Sci. Lett. 185, 25 (2001). 